\title{
Fuzzy based Reliable Cooperative Spectrum Sensing for Smart Grid Environment
}

\author{
Laila Nassef ${ }^{1}$, Reemah Al-Hebshi ${ }^{2}$ \\ Department of Computer Science \\ Faculty of Computing and Information Technology \\ King Abdulaziz University \\ Jeddah, Saudi Arabia
}

\begin{abstract}
The huge demand for spectrum has created immediate need to make available new licensed and/or unlicensed spectrum bands to satisfy the explosive growth of spectrum demands and to satisfy the quality of service requirements of diverse applications. Spectrum shortage and harsh environment have become a challenging bottleneck to achieve reliable communications in the smart grid. Cognitive radio is the emerging technology to achieve both spectrum and reliability awareness. Cooperative spectrum sensing takes advantage of spatial diversity to reduce the impact of receiver uncertainty. However, the harsh smart grid environments limit advantageous of cooperation due to variations of signal to noise ratio on which energy detection technique depends on. This paper proposes a reliable spectrum detection for a cluster based cooperative spectrum sensing in harsh smart grid environment, where cognitive cluster heads and a centralized cognitive radio based fusion center are deployed to solve both spectrum and reliability problems. The proposed fuzzy inference system is based on three fuzzy descriptors of energy difference, link quality, and local probability of detection. The results show the superiority of proposed fuzzy based fusion scheme to enhance accuracy of spectrum decision in harsh environment.
\end{abstract}

Keywords-Cognitive radio; wireless networks; cooperative spectrum sensing; reliable fusion; fuzzy inference system

\section{INTRODUCTION}

The recent technological developments in wireless technologies have enabled the deployment of the smart grid [1], which is the next generation of electrical power grid proposed to solve problems associated with traditional power grid [2]. The massive increase in diverse applications have resulted in exponential increase in demand for spectrum which have created immediate need to make available new licensed and/or unlicensed spectrum bands [3]. Wireless Sensor Networks (WSNs) have been considered as a promising technology to enhance capabilities of monitoring, control, and maintenance of the entire smart grid from power generation to transmission, distribution and consumption [4]. Different types of communication technologies are currently used to collect, aggregate, and analyze data collected from all sectors of the smart grid. However, the severe conditions of electrical power grid may adversely affect network reliability. Furthermore, WSNs are typically operate in the unlicensed Industrial, Scientific and Medical (ISM) frequency band which is already congested and overloaded with many other coexisting technologies. This increases interferences from neighboring networks and makes communication a challenging task due to spectrum scarcity and interference problems which could result in failure to establish and maintain reliable communications. There is need to sustainably support the continuously growing demand and to overcome both spectrum shortage and unreliable communications [4]. Cognitive radio (CR) technology allows the unlicensed Secondary Users (SUs) to opportunistically access the available licensed spectrum assigned to Primary Users (PUs) [5]. These underutilized licensed spectrum bands are assigned temporarily without the need to purchase the spectrum which offer reliable communications to ensure reliable communication in highly congested periods by transmitting data either on the original unlicensed channel or the additional licensed channel [6].

Spectrum Sensing is the most important function to detect the presence or absence of PUs at a certain location, at a given time, and in a specified frequency band. One of the spectrum sensing techniques is the transmitter detection which is further divided according to detection method into several methods, one of which is the energy detection [7]. Energy detection is the preferred technique for resource constraint nodes due to its low computational requirements compared to other techniques. Nodes in different spatial locations individually make local decision in a non-cooperative spectrum sensing by comparing the sensed energy with a predefined threshold to decide accordingly. However, due to the effect of propagation impairments, the Signal to Noise Ratio (SNR) of the received PU signal can be very low and individual SUs may not be able to distinguish between severely attenuated signal and vacant channel. Accurate spectrum sensing is important to mitigate the effect of environments and to guarantee reliable communications [8].

Cooperative Spectrum Sensing (CSS) has been proposed to enhance local spectrum decisions, where a group of SUs share their sensing reports with a Fusion Center (FC) either centralized or distributed to overcome receiver uncertainty by exploiting the spatial diversity [9]. In centralized CSS, the FC collects local spectrum sensing reports either as one-bit, quantized multi bits, or raw energy to identify spectrum occupancy, and broadcasts final global spectrum decision back to all nodes. Whereas in distributed CSS, each node shares spectrum reports with all of its neighbor nodes. CCS consumes energy in resource constraints nodes which cannot tolerate the heavy computations in distributed CSS. On the other hand, 
centralized CSS consumes large bandwidth and also consumes energy which reduce network’s lifetime [10].

CR based communications can be utilized in all sectors of the smart grid [11]. Reliable communication is urgently needed to sustainably solve spectrum scarcity problem, satisfy the ever increasing demand, and support the diverse applications [12]. However, the detection of available spectrum depends on multiple parameters which form a multi-objective optimization problem. Fuzzy logic can be utilized to solve this optimization problem that is difficult to be modeled using the mathematical methods. Fuzzy logic models are relatively simple and less computationally complex which make it suitable for resource constraints WSNs [13].

This paper proposes a Fuzzy Inference System (FIS) to enhance accuracy of cooperative spectrum detection in harsh smart grid environment. The harsh environment is prone to error and spectrum sensing reports will not be received correctly. To the best of our knowledge, none of the previous work have proposed a FIS to enhance detection accuracy in harsh smart grid environment. The contribution of this paper is the development of fuzzy logic based soft fusion strategy to accurately detect the opportunistic spectrum in harsh environment. The proposed system is based on three fuzzy descriptors of energy difference, channel condition, and local probability of detection.

The organization of this paper is as follows. Section II provides the related work. Section III describes the local spectrum sensing decisions in various environments. The performance indicators are also mathematically formulated. Section IV presents the cooperative hard fusion strategies in harsh environments. Section V proposes the FIS. The simulation results are demonstrated in Section VI. Finally, Section VII provides conclusion and future research work.

\section{RELATED WORK}

The increasing demand for more spectrum has motivated the development of CR based network to solve both spectrum scarcity and reliability problems facing the introduction of a wide range of new applications [14]. However, most of the previous work on spectrum sensing have assumed simplified propagation model in which received signal depends only on distance between transmitter and receiver. Additive White Gaussian Noise (AWGN) channel model for sensing channels was assumed while reporting channels were assumed to be dedicated error free channels. This model cannot accurately represent the real environments where propagation of electromagnetic signals suffers from propagation impairments which negatively impact the performance and present great challenge to reliability of wireless communications. The reliability of communication channels varies significantly from channel to channel and over time which impact the diverse applications which have stringent Quality of Service (QoS) requirements with respect to throughput, delay, and availability of the communications channels [15]. The random variation of the received signal makes spectrum sensing decision not accurate and nodes may fail to detect the spectrum due to presence of fading, shadowing, and time-varying nature of wireless channels. The randomness features of communication channels and variations in SNR causes uncertainty in sensing reports and makes it difficult to accurately achieve reliable spectrum detection decisions which could results in severe threat to reliability of network and sever threat to data integrity due to failure to satisfy spectrum demand by the diverse applications.

In Cluster-Based CSS, SUs perform spectrum sensing and send spectrum sensing reports to a Cluster Head $(\mathrm{CH})$ and aggregate sensing reports to FC to perform the energy consuming functions [16]. The FC combines the individual reports either by hard-decision or soft-data fusion to increase the accuracy of local spectrum sensing. In hard combining, sensing reports are received as a one-bit decision and combined using the counting rule of "l out of $N$ ", where "OR" rule, "AND” rule, and Majority rule are special cases [17]. Hard fusion decreases overhead on reporting channel, however the use of one energy threshold introduces information loss which degrade performance. On the other hand, soft fusion rules depend on raw sensed energy reports. The conventional soft combining schemes such as Square Law Combining (SLC), Maximal Ratio Combining (MRC), and Selection Combining (SC) enhance detection performance at a cost of increasing communication overhead. Nodes send their raw data to FC which consumes energy and bandwidth due to periodical transmission of raw data [18].

A tradeoff between overhead and detection performance is achieved by using a softened quantized multi-bit hard fusion [19]. Each SU quantizes its local observations into multiple decision regions to reduce overhead and achieve reliable sensing performance simultaneously. A quantization based multibit soft fusion rule is proposed in [20] to provide a compromise between sensing performance and control channel overhead. SUs use energy detector to observe the received signal level and compare it with quantization thresholds to estimate the multibit data. The data from all SUs are transmitted to FC to perform inverse quantization for the spectrum occupancy decision [21]. However, the more the number of bits, the more the overhead and the accurate the final global decision [22]. When the number of SUs is high, increasing the quantization bits provides no performance gain and quantized two bits energy report can be used. A softened two bits hard scheme that divides energy level into four quantization levels is developed in [23]. While in [24] a soft combining rule is proposed that assumed SNR is available at SUs. Similarly, a two-bit softened hard scheme is proposed in [25] for a cluster based CSS. One bit hard fusion is used based on SNR and one bit majority vote is used to combine the results.

Fuzzy logic can be utilized with CR based WSNs to produce the spectrum decision [26]. It is similar to natural language and can deal with uncertainty and imprecise knowledge for decision making. It allows phrases of real situations to feed mathematical models of reasoning. The statement truth of fuzzy logic depends on the degree of membership which have values between 0 and 1 . Fuzzy logic is used in [27] with three descriptors of spectrum utilization efficiency of the secondary user, of mobility, and its distance to the primary user. Fuzzy channel ranking estimation was presented in [28] using four descriptors of variability of the channel duration availability, duration of the channel idle time, 
channel condition and distance. Similarly, in [29] fuzzy based spectrum allocation was proposed to enhance spectrum efficiency based on three descriptors. IEEE 802.22 wireless regional area network (WRAN) is proposed for the smart grid to allow unlicensed broadband network to opportunistically access the unused TV white space [30]. Fuzzy logic is also applied to the IEEE 802.22 to prioritize channels in the backup and candidate channel list [31]. Finally, fuzzy logic was also proposed for IEEE 802.22 WRAN in [32] to select channels based on required QoS using throughput, latency and reliability.

\section{LOCAL DECISION IN VARIOUS ENVIRONMENTS}

The FC is responsible of coordinating the spectrum availability and providing the list of available spectrum to be scanned and utilized. SUs sense the spectrum and transmit their local spectrum decisions about spectrum availability to their corresponding CR based Cluster Head $(\mathrm{CH})$. The network is clustered into $c$ clusters with cluster 1 , cluster 2 , and cluster $\mathrm{j}$, each has cluster members of $k_{1}, k_{2}, . ., k_{c}$ respectively, where $1 \leq j \leq k_{c}$. The $\mathrm{CHs}$ collect sensing reports, schedule and distribute the spectrum sensing tasks among the cluster members. Upon joining a cluster, $j$, a $S U_{i}$ becomes a cluster member defined as $S U_{i}^{j}$. The sensing reports from all cluster heads $\mathrm{CH}^{j}$ are collected, to make the spectrum sensing decision, and this decision is propagated back to SUs to access the available spectrum.

The functionality of spectrum sensing is implemented at both physical and MAC layers [33]. Physical layer detects PU's signal using energy detection and MAC focuses on time to sense and channels to sense [34]. CHs schedule and transmit sensing reports in a Time Division Multiple Access (TDMA) where total sensing period is divided into a number of sensing slots, each has a duration of $T_{t_{i}}$ that is divided into two subslots: a sensing sub-slot of $T_{s_{i}}$ and a reporting sub-slot of $T_{r_{i}}$. All SUs sense the channels in the assigned time slot and then forward sensing report over the reporting channel in the scheduled reporting time slots [35]. The spectrum sensing may behave as binary with hypothesis testing problem, if there are no activities on the channel, it is considered as a null hypothesis $H_{0}$ or otherwise it is considered as busy channel $H_{1}$.

All $S U S$ forward their sensing reports to FC about each channel. Without loss of generalities, the following is for one channel, where the local spectrum decision is decided based on local energy threshold, sensing parameters, and environment. The propagation is modeled as a simplified AWGN non fading, or realistic environments lognormal shadowing with no channel errors, or harsh environment with errors.

\section{A. Non-Fading AWGN Environments}

The spectrum sensing in AWGN assumes sensing channels to be error free with AWGN [33]. For each cluster member $S U_{i}^{j}$, the received signal in the $\tau^{t h}$ sensing time is defined as $y_{i}(\tau)$, where $i=1, \ldots, k_{c}$, is formulated as:

$\begin{cases}y_{\mathrm{i}}(\tau)=n_{\mathrm{i}}(\tau) & : \mathrm{H}_{0} \\ y_{\mathrm{i}}(\tau)=h_{\mathrm{pi}} \cdot x_{\mathrm{p}}(\tau)+n_{\mathrm{i}}(\tau): & \mathrm{H}_{1}\end{cases}$ where $\tau=1, \ldots, \mathcal{S}$, where $\mathcal{S}$ is the number of received samples within the spectrum sensing period $T_{s}$ such as $\mathcal{S}=T_{s} f_{s}$. The PU signal sensed over the sensing channel $x_{p_{i}}(\tau)$ has a carrier frequency of $f_{s}$ with zero mean and variance of $\sigma_{p_{i}}$. The AWGN noise signal $n_{i}(\tau)$ has zero means and variance of $\sigma_{n_{i}}$. Both PU's signal $\mathcal{X}_{p_{i}}(\tau)$ and noise signal $n_{i}(\tau)$ are assumed to be independent and identically distributed random processes. $h_{p i}$ is the channel fading coefficient. When number of samples " $\mathcal{S}$ " is large enough, the Probability Distribution Function (PDF) of $\mathcal{Y}_{i}$ follows a central chi square distributed under hypothesis $H_{0}$ and a non-central chi-square distributed with $\mathcal{N}$ degree of freedom under hypothesis $H_{1}$. Then the observed sensed energy statistics $E_{i}$ can be approximated by the Gaussian distributions and formulated as:

$E_{i}=\frac{1}{\mathcal{S}} \sum_{\tau=1}^{\mathcal{S}}\left|y_{i}(\tau)\right|^{2}$

The local hard decision $u_{i}$ of the individual SU is computed by comparing its received energy $E_{i}$ with a predetermined threshold $\lambda_{t h}$ to decide on hypothesis $\mathcal{H}_{1}$ and $\mathcal{H}_{0}$ that is formulated as:

$u_{i}=\left\{\begin{array}{l}\mathcal{H}_{1}: E_{i} \geq \lambda_{t h} \\ \mathcal{H}_{0}: \text { otherwise }\end{array}\right.$

The performance indicators are evaluated to know whether the sensed value is generated under hypothesis $\mathcal{H}_{0}$ or hypothesis $\mathcal{H}_{1}$ which represent idle and occupancy state the PU. The probability of detection $\left(P_{d}\right)$ is the probability that decision is $\mathcal{H}_{1}$ when $\mathcal{H}_{1}$ is true which is formulated as:

$P_{d}=P\left[\mathcal{H}_{1} \mid \mathcal{H}_{1}\right]=P\left[E_{i}>\lambda_{\text {th }} \mid \mathcal{H}_{1}\right]$

The probability of false alarm $\left(P_{f a}\right)$ is the probability that decision is $\mathcal{H}_{1}$ but is true which is formulated as.

$P_{f a}=P\left[\mathcal{H}_{1} \mid \mathcal{H}_{0}\right]=P\left[E_{i}>\lambda_{t h} \mid \mathcal{H}_{0}\right]$

$P_{f a}$ is an indicator of the spectrum utilization, where a high value of $P_{f a}$ means less spectrum utilization and lower value of $P_{f a}$ means higher spectrum utilization. Both higher $\left(P_{d}\right)$ and lower $\left(P_{f a}\right)$ provide more protection for the PUs and improved spectrum utilization. As $E_{i}$ follows a Gaussian distribution with zero mean and variance of $\sigma_{n}^{2}$, then performance is approximated as follows:

$$
\begin{aligned}
& E_{i} \mid \mathcal{H}_{1}=P_{d} \sim \mathcal{N}\left((1+\gamma) \sigma_{n}^{2}, \frac{2(1+\gamma)^{2} \sigma_{n}^{4}}{\mathcal{S}}\right) \\
& E_{i} \mid \mathcal{H}_{0}=P_{f a} \sim \mathcal{N}\left(\sigma_{n}^{2}, \frac{2 \sigma_{n}^{4}}{\mathcal{S}}\right)
\end{aligned}
$$

where $\gamma$ is SNR. The performance indicators in AWGN environment are computed as follows:

$$
\begin{aligned}
& P_{d}=E_{i} \mid \mathcal{H}_{1}=Q\left(\left[\frac{\frac{\lambda_{t h}}{\sigma_{n}^{2}}}{(1+\gamma)}-1\right] \sqrt{\frac{T_{s} f_{s}}{2}}\right) \\
& P_{f a}=E_{i} \mid \mathcal{H}_{0}=Q\left(\left[\frac{\lambda_{t h}}{\sigma_{n}^{2}}-1\right] \sqrt{\frac{T_{s} f_{s}}{2}}\right)
\end{aligned}
$$


where $\mathrm{Q}($.$) is the complementary distribution function of$ the standard Gaussian function.

\section{B. Error Free Fading Environment}

The smart grid have noise, interferences, and multipath propagation effects [36], and the received SNR at different SUs is not the same and can be computed as follows.

$$
S N R_{i}^{j}=\gamma_{i}^{j}=\frac{h_{p i}(\tau) x_{p i}(\tau)}{\sum_{l=1, l \neq i}^{k_{c}} h_{l i}(\tau) x_{l i}(\tau)+\sigma_{l}^{2}}
$$

where there is 1 node index and $l<k_{c}$, $\sum_{l=1, l \neq i}^{k_{c}} h_{l i}(\tau) X_{l i}(\tau)$ is the accumulated interfering signals coming from $l$ nodes. For the error free fading Environments (channel error probability $P_{e}=0$ ), the sensing channels will have variable channel fading coefficient $h_{p i}$ and consequently have variable $S N R$. The average probability of detection $\bar{P}_{d, i}$ will be computed as

$\bar{P}_{d, i}=\int_{0}^{\infty} Q\left(\left[\frac{\frac{\lambda_{t h}}{\sigma_{i n}^{2}}}{\left(1+\gamma_{i}\right)}-1\right] \sqrt{\frac{T_{s} f_{s}}{2}}\right) f\left(\gamma_{i}\right) d \gamma_{i}$

where $f\left(\gamma_{i}\right)$ is the Probability Distribution Function (PDF) of $\gamma_{i}$. Under $H_{0}$ case, the $P_{f a}$ is independent of $\gamma_{i}$ and the average $\bar{P}_{f a, i}$ is the same for all $S U_{i}$ as computed in equation (9). Then the PDF of $\gamma_{i}$ can be approximated.

$f\left(\gamma_{i}\right)=\sqrt{\frac{\alpha_{i}}{2 \pi \gamma_{i}}} \frac{1}{\gamma_{i}} \exp -\frac{\alpha_{i}\left(\gamma_{i}-\beta_{i}\right)^{2}}{2 \beta_{i}{ }^{2} \gamma_{i}} ; \gamma_{i} \geq 0$

where $\beta_{i}$ is the expectation of $\gamma_{i}$ i.e., $\beta_{i}=\boldsymbol{\varepsilon}\left[\gamma_{i}\right]$, and $\alpha_{i}$ is the shape parameter. In lognormal distribution, the relation between the parameters $\left\{\alpha_{i}, \beta_{i}\right\}$ and $\left\{\mu_{i}, \sigma_{i_{d B}}\right\}$ is specified as follow:

$\beta_{i}=\exp \left(\frac{\mu}{\Psi}, \frac{\sigma_{i d B}^{2}}{2 \psi^{2}}\right), \quad \alpha_{i}=\frac{\beta_{i}}{\exp \left(\frac{\sigma_{d B}^{2}}{\psi^{2}}\right)-1}$

where $=\frac{10}{\ln (10)}, \mu$ is mean, and $\sigma_{i}$ is the standard deviation of the $S N R_{i}$ in logarithmic scale $\left(10 \log 10 \gamma_{i}\right)$. Then $Q(x)$ function is computed as $\frac{1}{2} \operatorname{erfc}\left(\frac{x}{\sqrt{2}}\right)$, where $\operatorname{erfc}($. ) represents complementary error function and defined as follows:

$\operatorname{erfc}(x)=1-\frac{2}{\sqrt{\pi}} \sum_{k=0}^{\infty} \frac{(-1)^{k} t^{2 k+1}}{2^{k+\frac{1}{2}} k !(2 k+1)}$

In this case, $Q(x)$ function is computed as

$Q(x)=\frac{1}{2} \frac{1}{\sqrt{\pi}} \sum_{k=0}^{\infty} \frac{(-1)^{k} t^{2 k+1}}{2^{k+\frac{1}{2}} k !(2 k+1)}$

Where $t=\left[\frac{\frac{\lambda_{t h}}{\sigma_{i}^{2}}}{\left(1+\gamma_{i}\right)}-1\right] \sqrt{\frac{T_{s} f_{s}}{2}}$

The average probability of detection $\bar{P}_{d, i}$ at different $S U_{i}$ is a function of $\gamma_{i}$ and is formulated as:

$\bar{P}_{d, i}=\sqrt{\frac{\alpha_{i}}{8 \pi}} \int_{0}^{\infty} \gamma_{i}^{-3 / 2} \exp \left[-\frac{\alpha_{i}\left(\gamma_{i}-\beta_{i}\right)^{2}}{2 \beta_{i}{ }^{2} \gamma_{i}}\right] d \gamma_{i}-$ $\sqrt{\frac{\alpha_{i}}{2 \pi^{2}}}\left(\frac{T_{s} f_{s}}{2}\right)^{2 k+1} \sum_{k=1}^{\infty} \frac{(-1)^{k}}{2^{k}+\frac{1}{2}(2 k+1) k !} \times$

$\int_{0}^{\infty}\left(\frac{\lambda_{t h} / \sigma^{2}}{1+\gamma_{i}}-1\right)^{2 k+1} \gamma_{i}^{-3 / 2} \exp \left[-\frac{\alpha_{i}\left(\gamma_{i}-\beta_{i}\right)^{2}}{2 \beta_{i}{ }^{2} \gamma_{i}}\right] d \gamma_{i}$

For a target performance and if each cluster is assumed to have the same channel gain, the performance indicators can be approximated as follow:

$\bar{P}_{d, i}=\operatorname{Pr}\left\{u_{i}=1 \mid \mathcal{H}_{1}\right\}, \bar{P}_{d, i}=\bar{P}_{d} ; \forall i$

$\bar{P}_{f a, i}=\operatorname{Pr}\left\{u_{i}=1 \mid \mathcal{H}_{0}\right\}, \bar{P}_{f a, i}=\bar{P}_{f a} ; \forall i$

$\bar{P}_{m d, i}=\operatorname{Pr}\left\{u_{i}=0 \mid \mathcal{H}_{1}\right\}, \bar{P}_{m d, i}=\bar{P}_{m d} ; \forall i$

where $\bar{P}_{m d}=1-\bar{P}_{d}$ is the probability of miss detection.

\section{Harsh Environment}

Due severe noise and shadow fading in sensing channels in each cluster, the interference increases packet loss rate, which increases demand due packet retransmissions and consequently deteriorate QoS due increase in retransmission. This causes errors and the performance indicators should be modified to include the channel error probability $P_{e}$ as follows:

$\bar{P}_{f e}=\bar{P}_{f a}\left(1-P_{e}\right)+\left(1-\bar{P}_{f a}\right) P_{e}$

$\bar{P}_{m d e}=\bar{P}_{m d e}\left(1-P_{e}\right)+\left(1-\bar{P}_{m d e}\right) P_{e}$

\section{COOPERATIVE HaRd Fusion IN HARSh ENVIRONMENT}

Local spectrum sensing performs poorly in harsh environment. CSS exploits the spatial diversity from multiple spectrum sensing nodes, where each $S U_{i}$ submits a one bit local decision to the $C H^{j}$ through the reporting channels to aggregate these reports to the FC to combine these reports using hard fusion strategies or soft fusion strategy if reports have raw data. CSS improves detection performance where nodes in different spatial locations share their sensing reports with the FC to overcome uncertainties of individual decision. Either hard or soft fusion strategies can be used [37]. Local binary decisions are transmitted to FC to combine these sensing reports to evaluate performance indicators based on OR, AND, or majority voting strategies [38]. Similar to the local decision, the final CSS decision is measured based on both $P_{d}$ and $P_{f a}$.

Under 'AND' rule FC decides the presence of PU when all the SU's detects the PU signal.

$P_{D}{ }^{A N D}=\prod_{i=1}^{k_{c}} P_{d}, P_{F A}{ }^{A N D}=\prod_{i=1}^{k_{c}} P_{f a}$

Under 'OR' rule, at least one of the SU's involved in the sensing decides the presence of the PU.

$P_{D}{ }^{O R}=1-\prod_{i=1}^{k_{c}}\left(1-P_{d_{i}}\right)$,

$P_{F A}{ }^{O R}=1-\prod_{i=1}^{k_{c}}\left(1-P_{f a_{i}}\right)$

Under majority fusion rule (half voting rule), the cooperative probabilities can be simplified and formulated 
using the normal Binomial distribution (instead of PoissonBinomial) to become:

$$
\begin{aligned}
& Q_{d}^{M A J}=\sum_{l=i}^{k_{c}}\left(\begin{array}{c}
k_{c} \\
l
\end{array}\right) P_{d}^{l}\left(1-P_{d}\right)^{k_{c}-l} \\
& Q_{f a}{ }^{M A J}=\sum_{l=i}^{k_{c}}\left(\begin{array}{c}
k_{c} \\
l
\end{array}\right) P_{f}^{l}\left(1-P_{f}\right)^{k_{c}-l}
\end{aligned}
$$

In harsh environment, performance indicators are modified to include $P_{e}$ as follow:

$$
\begin{aligned}
& Q_{d e}{ }^{\text {MAJORITY }}=\sum_{l=i}^{k_{c}}\left(\begin{array}{c}
k_{c} \\
l
\end{array}\right) P_{d e}{ }^{l}\left(1-P_{d e}\right)^{k_{c}-l} \\
& Q_{f a e^{\text {MAJORITY }}}=\sum_{l=i}^{k_{c}}\left(\begin{array}{c}
k_{c} \\
l
\end{array}\right) P_{f a e}{ }^{l}\left(1-P_{f a e}\right)^{k_{c}-l} \\
& Q_{m d e}{ }^{\text {MAJORITY }}=1-\sum_{l=i}^{k_{c}}\left(\begin{array}{c}
k_{c} \\
l
\end{array}\right) P_{m d e}{ }^{k_{c}-l}\left(1-P_{m d e}\right)^{l}
\end{aligned}
$$

\section{PRoposed FuZZY INFERENCE SySTEM}

In harsh environments, depending on energy statistic as the only parameter to detect $P U$ is not enough to correctly detect the spectrum, as the received energy samples are corrupted by propagation impairments which cause performance degradation at low values of SNR. Fuzzy logic can deal with uncertainty in data by converting imprecise data into precise data. The FIS utilizes four steps of fuzzification, fuzzy inference engine, fuzzy rule base, and defuzzification as depicted in Fig. 1.

The fuzzification converts crisp values into linguistic variables to determine the membership function in which the fuzzy rule IF-THEN is applied to form the new outputs set. Three antecedents or descriptors are combined compute the spectrum detection decision. The first descriptor is energy difference, the second is the channel condition or SNR, and third is the local probability of detection which is directly computed. Each descriptor is assigned three linguistic variables to compute the reliable probability of detection. The defuzzifier computes the crisp output from the rule output sets.

The FIS computes reliable spectrum decision based on these three descriptors. Each is divided into three levels of linguistic values to reflect different degree of membership of input linguistic variables. These values are set after analyzing their range. The Fuzzification converts crisp values into linguistic variables. The rule base consists of a number logic rules in the form IF-THEN statements, where 'IF' part of the rule is called 'antecedent' and the 'THEN' part of the rule is called 'consequent'. These rule clauses depend on the number of linguistic variables and membership functions to find optimal solutions. The three descriptors are computed as follows.

\section{A. Energy Matrix}

The energy matrix is constructed from the received reports and the absolute energy difference $D I F F{ }_{i}^{j}$ for each $S U_{i}^{j}$ from the average energy for all other $S U S$ in each cluster $j$ is computed by neglecting the $S U_{i}^{j}$ result in each sensing interval to evaluate impact of not including this particular $S U_{i}^{j}$ in the result. The energy difference matrix $D I F F_{i}^{j}$ for each node $i$ in cluster $j$ is formulated as

$\operatorname{DIFF}_{i}^{j}=\left|E_{i}^{j}-\frac{1}{k_{c}} \sum_{j=1}^{k_{c}} E_{i}^{j}(j=1,2, \ldots, c)\right|$

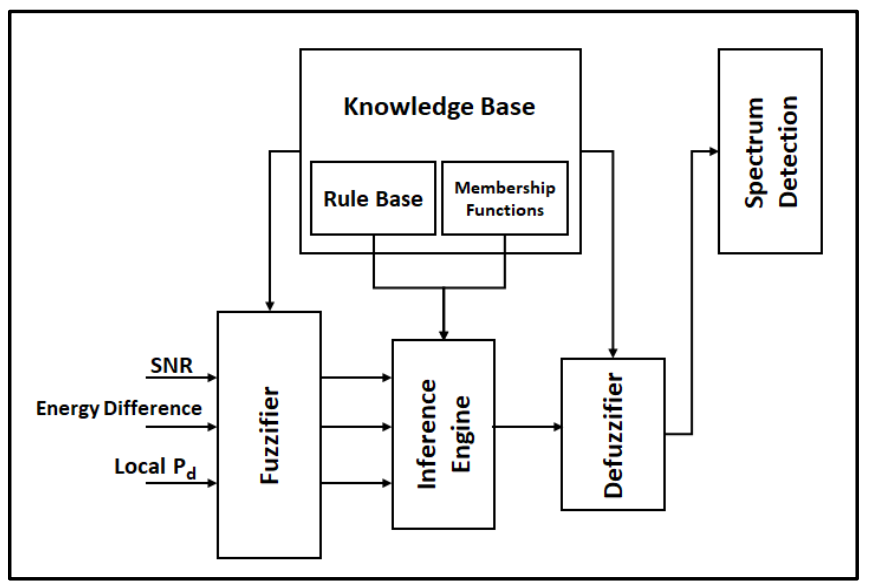

Fig. 1. Proposed Fuzzy Inference System.

The total number of $S U_{i}^{j}$ in cluster $\mathrm{j}$ is $k_{j}$, where $k_{1}, k_{2}$, $\ldots, k_{c}$ and $1 \leq j \leq c$ means that $k_{1} S U s$ in Cluster $1, k_{2} S U S$ in Cluster $2, \ldots k_{c} S U S$ in cluster "c", respectively. The average value of energy reports of all other SUs is evaluated by keeping away the sensing report of the $S U_{i}$ out of the average cluster's energy. This value is standardized to get its consistent dimensions as follows:

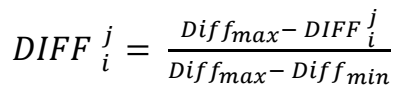

Energy difference to cluster average ( $\operatorname{Diff}{ }_{j}^{i}$ ) is the Absolute differences of the sensing energy of $S U_{i}^{j}$ with the average sensing energy provided by all other SUs in each cluster. It has three linguistic values of Low, Medium, and High.

\section{B. Channel Condition Matrix}

The SNR, computed as $\left(\gamma_{i}^{j}\right)$, is standardized to get its consistent dimensions as follows:

$\gamma_{i}^{j}=\frac{\gamma_{\max }-\gamma_{i}^{j}}{\gamma_{\max }-\gamma_{\min }}$

$\gamma_{i}^{j}$ has three linguistic values of Poor, Adequate, and Excellent.

\section{Probability of Detection Matrix}

The local probability of detection for each $S U_{i}^{j}$ is defined as $P_{d_{i}}$ which has three linguistic values of Low, Medium, and High.

\section{Reliable Probability of Detection}

The output linguistic values that represent reliable decision for each $S U_{i}^{j}$ and defined as $P_{d_{i}}^{j}$ has seven linguistic values of Very High, High, Med High, Mid, Mid Low, Low, Very Low. Since there are three inputs parameters, each of them have three levels, a total of 27 fuzzy rules are used. The output is defuzzified to convert fuzzy output into crisp values again which is the actual output of the system or the reliable $P_{d, i}^{j}$ spectrum sensing decision. 


\section{E. Membership Functions}

Many types of membership functions can be used to cover the complete input and output range, from which triangle and trapezoidal membership functions are the most useful types due to their simplicity to determine their degrees of membership. The middle levels of the three inputs re represented by triangle membership functions and the other levels are represented by trapezoidal membership functions. The seven-output linguistic variable are represented by triangular function as shown in Fig. 2. The two extreme IFELSE statement rules fall between these two cases:

Case (1): If Diff ${ }_{j}^{i}$ is high, $\gamma_{i}^{j}$ is poor, and $P_{d, i}^{j}$ is low then Reliable $P_{d, i}^{j}$ is verylow.

Case (2): If $\operatorname{Diff}{ }_{j}^{i}$ is low, $\gamma_{i}^{j}$ is excellent, and $P_{d, i}^{j}$ is high then Reliable $P_{d, i}^{j}$ is veryhigh.

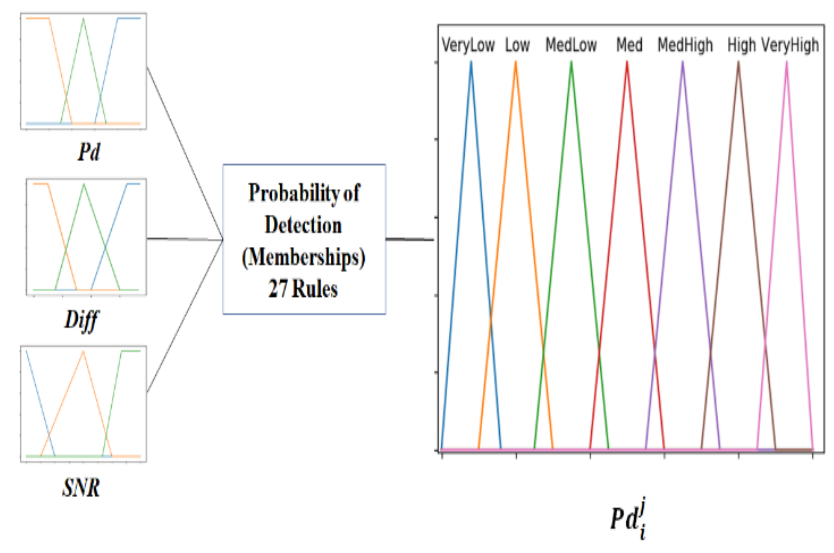

Fig. 2. The Input and Output Membership Functions.

\section{SIMULATION AND RESUlt DisCUSSIONS}

The cluster architecture is naturally suitable for all sectors of the smart grid, one of which is the Home Area Networks (HANs). HANs consist of a large number of smart appliances that communicate with utility to support various smart grid applications. This environment is simulated with all of the required simulation parameters selected based on this specific environment. The sensing reports are collected and transmitted to the corresponding $\mathrm{CH}$ which aggregate these reports to the FC to compute reliable spectrum decision based on the proposed FIS. The range of the inputs and outputs of the three descriptors are selected based on experience acquired from running several simulations. The numerical values are then correlated with linguistic variables describing their degree of belonging to each of the three variables. A rule table, in the form of if-then statements, is used to represent the fuzzy rules to generate the values after defuzzification is done on the output.

\section{A. Simulation Environment}

The network nodes are assumed to be static SUs and are deployed within an area of $100 \mathrm{mx} 100 \mathrm{~m}$. SUs are assumed to have a single interface that can switch among $C$ traffic channels accessed opportunistically, along with the predefined common control channel. These SUs coexist with M PUs that can appear on C channels. The SUs can opportunistically access the available licensed channels only when PU is not active. The PU activity is modelled as independent and identically distributed random process with busy and vacant probabilities. Nodes are assumed to sense highly correlated sets of channels. Each cluster is assumed to have only 5 available channels to generate congestion. The propagation parameters are selected to give a high impact of environment. The traffic parameters are also selected based on data traffic load found in most HAN applications, which have a periodic traffic that is generated with constant bit rate (CBR) packets and have a rate of one data packet every 5 seconds to overload the network. Each packet has a size of 512 bytes, which corresponds to most smart grid applications. In addition, the data rate is set to the maximum data rate $250 \mathrm{kbps}$. The common simulation parameters are presented in Table I.

TABLE I. COMMON SIMULATION PARAMETERS

\begin{tabular}{|l|l|l|l|}
\hline Parameter & Values & Parameter & Values \\
\hline Fetwork radius & $100 \mathrm{~m}$ & $\begin{array}{l}\text { Target Probability of } \\
\text { false alarm } P_{f a}\end{array}$ & 0.1 \\
\hline Unlicensed Band & IEEE802.15.4 & $\begin{array}{l}\text { Channel Error } \\
\text { probability } P_{e}\end{array}$ & 0.9 \\
\hline Number of SUs & 100 & $\begin{array}{l}\text { Target Probability of } \\
\text { Setection } P_{d}\end{array}$ & 0.3 \\
\hline SU coverage radius & $35 \mathrm{~m}$ & $\begin{array}{l}\text { Sensing report duration } \\
T_{s}\end{array}$ & $1 \mathrm{msec}$ \\
\hline Number of channels & 5 & Sampling duration $\tau$ & $0.1 \mathrm{msec}$ \\
\hline Path loss exponent & 4.2 & Number of samples $(\mathcal{S})$ & 500 \\
\hline Shadowing Variance & 4.0 & PU coverage radius & $50 \mathrm{~m}$ \\
\hline Receiver sensitivity & $-85 \mathrm{dBm}$ & Number of PU & 5 \\
\hline licensed Band & IEEE802.11 & Frequency band & $2.4 \mathrm{GHz}$ \\
\hline
\end{tabular}

\section{B. Simulation Results and Analysis}

The impact of various environments, for different values of $S N R$, on the probability of detection for various fusion rules of OR, AND, Majority rule, and the proposed fuzzy fusion under both AWGN and harsh environment are presented in Fig. 3. Generally, the cluster based CSS enhances performance over local decision in harsh environment. The OR logic rule with and without error shows good performance compared to AND rule and Majority rule. The proposed fuzzy fusion has the highest detection probability in AWGN environment than other fusion rules due to enhancement in decision accuracy. However, the harsh environment has caused severe threat to reliability of sensing reports and threaten network operation due to failure to find any available spectrum. This has degraded performance in all schemes and reduced detection accuracy at lower values of SNR. The developed FIS was effective to enhance spectrum detection using three fuzzy descriptors of sensing node energy, channel condition, and local sensing decision. At the sensing node, lower values of $S N R$ indicate unreliable sensing environment which introduce errors and degrade the reliability of sensing reports.

The performance of all fusion rules is degraded significantly under lower values of $S N R$ which made spectrum 
sensing difficult due to incorrect reception of sensing reports and inability to differentiate signal from noise. The degradation in detection probability for all schemes is due to inaccurate sensing reports that cause uncertainty and render the energy detector useless. For a target $P_{f a_{i}}$, as $S N R$ increases, higher values of $P_{d_{i}}$ is achieved as nodes are able to properly detect spectrum and provide more reliable sensing reports. The proposed fuzzy fusion has better performance than AND and Majority fusion rules due to enhancement in decision accuracy even at smaller values of $S N R$.

The harsh environment which is prone to error and sensing reports may not be received correctly have greatly affected all schemes and decreased the probability of detection. The fuzzy fusion was able to maintain the performance to a certain limit after which it could not compensate for errors introduced in environments. However, the OR logic fusion shows relatively good performance than the proposed fuzzy fusion and was able to achieve good performance even in harsh environment.

The probability of detection against probability of false alarm is presented in Fig. 4, which the proposed fuzzy fusion is compared with both majority fusion and local detection under both AWGN and harsh environments. The proposed fuzzy fusion in AWGN is shown for comparison which has the highest detection probability than other fusion rules due to enhancement in decision accuracy. The proposed FIS has enhanced the detection probability compared to other schemes and it is able to provide a sustainable solution to solve both spectrum scarcity and reliability problems in harsh environments. The reliable fuzzy based spectrum detection has accurately mitigated the impact of unreliable environment and has achieved more efficient and robust detection than other schemes. However, harsh environment is prone to error and sensing reports may not be received and this causes lower performance of the proposed fuzzy fusion under harsh environment as compared to the AWGN case.

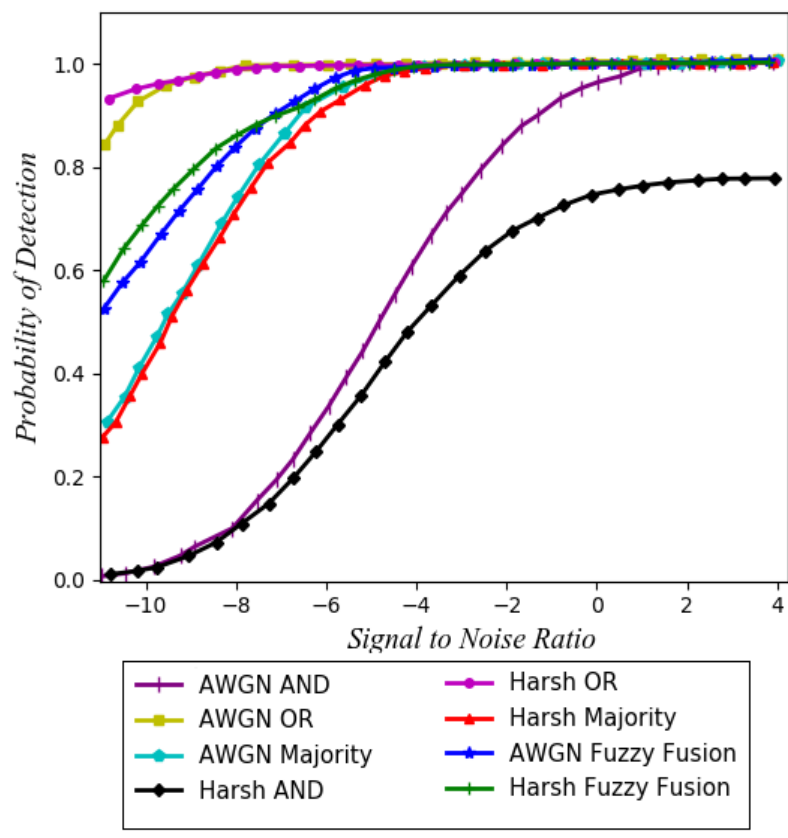

Fig. 3. The Probability of Detection Against Signal to Noise Ratio.

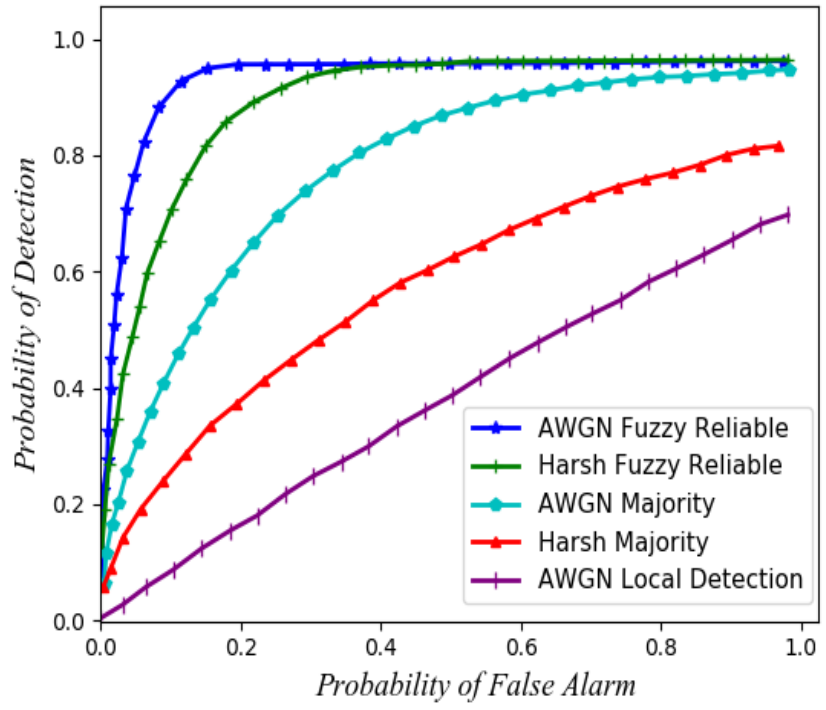

Fig. 4. The Probability of Detection Against Probability of False Alarm.

Fig. 5 plots the probability of miss detection against probability of false alarm $P_{f a}$ for different SNR. At lower SNR, performance deteriorates mainly because the energy detection method is based on received signal's energy and consequently higher noise levels at receiver greatly impact performance indicators. The probability of miss detection increases with the decrease in SNR and the harsh environment which introduces errors that leads to incorrect reception of sensing reports. At lower values of SNR, the probability of miss detection reaches 0 only when probability of false alarm is close to 1 . However, for better values of SNR, lower values of probability of miss detection is achieved at lower values of probability of false alarm.

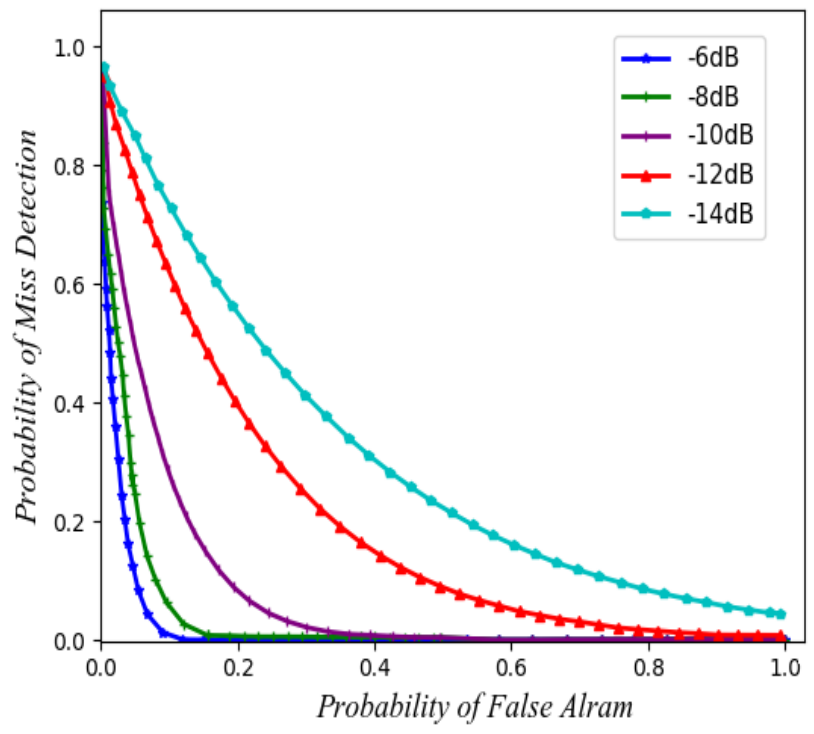

Fig. 5. Probability of Miss Detection Against Probability of False Alarm.

The lower values of probability of miss detection is achieved only at higher SNR. However, at lower SNR, nodes cannot recognize the signal and could identify the signal as a noise and consequently it will consider channel as vacant. The 
harsh environment is subjected to errors that impact probability of false alarm $P_{f a}$ which occurs if an idle channel is detected as busy and may lead to a potentially wasted opportunity for the SU to transmit. Also it impact the probability of miss detection $P_{m d}$ which occurs when a busy channel is detected as idle and could potentially lead to a collision or interference with the PU, leading to wasted transmissions for both PU and SU.

\section{CONCLUSION AND FUTURE WORK}

This paper proposes the use of a fuzzy inference system to enhance spectrum detection in a cluster based cooperative spectrum sensing in harsh environment. The proposed fuzzy based soft fusion is simulated and evaluated using the probability of detection and the probability of false alarm. The results indicated that the proposed FIS have significantly outperforms other fusion rules in harsh environment. It was effective to enhance probability of detection and provide more robust fusion decision by incorporating environment in decision making.

In this paper, the QoS was assumed to be satisfied by only accessing the available spectrum, however, various applications demand different data rates and use different data size with various transmission times which need to adapt to the PU activities. The PU's activities was fixed and the proposed FIS urgently needs to consider PU activities, sort the available channels based on their quality, and allocate available spectrum based on QoS requirements. Further maximization is expected by adapting sensing parameter to react to environment accordingly. However, these sensing parameters should be optimized along with other parameters to satisfy the QoS requirements of diverse applications. An immediate future work will be the development of this complex problem.

\section{REFERENCES}

[1] Melike Yigit, V. Cagri Gungor, E. Fadel, L. Nassef, N. Akkari and I. F. Akyildiz, "Channel-Aware Routing and Priority-Aware Multi-Channel Scheduling for WSN-Based Smart Grid Applictions", Journal of Network and Computer Applications, vol. 71, pp. 50-58, 2015. https://doi.org/10.1016/j.jnca.2016.05.015.

[2] Yin XC, Liu ZG, Nkenyereye L and Ndibanje B, "Toward an Applied Cyber Security Solution in IoT-Based Smart Grids: An Intrusion Detection System Approach”, Sensors (Basel), vol. 19, no. 20, 2019. DOI: $10.3390 / \mathrm{s} 19224952$.

[3] Laila Nassef, Remah Elhebshi and Linta Jose, "Evaluating Performance of Wireless Sensor Network in Realistic Smart Grid Environment", International Journal of Wireless \& Mobile Networks, vol. 10, no. 3, pp. 27-36, 2018. DOI: 10.5121/ijwmn.2018.10303.

[4] Etimad Fadel, V.C. Gungor, Laila Nassef, Nadine Akkari, M.G. Abbas Malik, Suleiman Almasri and Ian F. Akyildiz, "A Survey on Wireless Sensor Networks for Smart Grid”, Computer Communications, vol. 71, pp. 22-33, 2015. https://doi.org/10.1016/j.comcom.2015.09.006.

[5] Ian F. Akyildiz, Won-Yeol Lee, Mehmet C. Vuran and Shantidev Mohanty, "Next Generation/Dynamic Spectrum Access/Cognitive Radio Wireless Networks: A Survey”, Computer Networks, vol. 50, no. 13, pp. 2127-2159, 2006. https://doi.org/10.1016/j.comnet.2006.05.001.

[6] Xin-Lin Huang, Xiao-Wei Tang and Fei Hu, "Dynamic Spectrum Access for Multimedia Transmission Over Multi-User, Multi-Channel Cognitive Radio Networks”, IEEE Transactions on Multimedia, pp. 201 - 214, 2020. DOI: 10.1109/TMM.2019.2925960.

[7] James Adu Ansere, Guangjie Han, Hao Wang and Celimuge Wu, “A Reliable Energy Efficient Dynamic Spectrum Sensing for Cognitive Radio IoT Networks”, IEEE Internet of Things Journal, vol. 6, no. 4, pp. 6748 - 6759, 2019. DOI: 10.1109/JIOT.2019.2911109.
[8] Yu Qiao, Alex Liu and Lejun Zhang, "EESS: An Energy-Efficient Spectrum Sensing Method by Optimizing Spectrum Sensing Node in Cognitive Radio Sensor Networks”, Hidawi Journal, 2018. https://doi.org/10.1155/2018/9469106.

[9] Xue Zhang, Xiaozhu Liu, Hooman Samani and Brian Jalaian, "Cooperative Spectrum Sensing in Cognitive Wireless Sensor Networks”, International Journal of Distributed Sensor Networks, 2015. https://doi.org/10.1155/2015/170695.

[10] Laila Nassef , Reemah El-Habshi and Linta Jose, "Clustering-Based Routing For Wireless Sensor Networks In Smart Grid Environment”, International Journal of Advanced Smart Sensor Network Systems (IJASSN), vol. 8, no. 1, 2018. DOI:10.5121/ijassn.2018.8301.

[11] E. Fadel, M. Faheem, V. Gungor, L. Nassef, N. Akkari, M. A. Malik, S. Almasri and I. F. Akyildiz, "Spectrum-Aware Bio-Inspired Routing in Cognitive Radio Sensor Networks for Smart Grid Applications", Computer Communications, vol. 101, pp. 106-120, 2017. https://doi.org/10.1016/j.comcom.2016.12.020.

[12] Dr. Laila Nassef and Reemah Alhabshi, "Energy Efficient Fuzzy Based Clustering for Cognitive Radio Wireless Sensor Networks", International Journal of Electrical \& Computer Sciences IJECS-IJENS, vol. 18, no. 5, 2018, ISSN: 2077-1231 (Online), 2227-2739 (Print).

[13] Dost Muhammad Saqib Bhatti, Nasir Saeed and Haewoon Nam, "Fuzzy C-Means Clustering and Energy Efficient Cluster Head Selection for Cooperative Sensor Network”, Sensor, vol. 16, no. 9, 2016. DOI: 10.3390/s16091459.

[14] Dina Tarek, Abderrahim Benslimane, M. Darwish and Amira M. Kotb, "Survey on Spectrum Sharing/Allocation for Cognitive Radio Networks Internet of Things", Egyptian Informatics Journal, 2020. https://doi.org/10.1016/j.eij.2020.02.003.

[15] Muhammad Waqas Khan and Muhammad Zeeshan, "QoS-Based Dynamic Channel Selection Algorithm for Cognitive Radio Based Smart Grid Communication Network”, Ad Hoc Networks, vol. 87, pp. 61-75, 2019. https://doi.org/10.1016/j.adhoc.2018.11.007.

[16] Laila Nassef and Reemah Alhebshi, "Secure Spectrum Sensing In Cognitive Radio Sensor Networks: A Survey”, International Journal of Computational Engineering Research (IJCER), vol. 6, no. 3, p. $2250-$ 3005, 2016.

[17] Pankaj Verma and Brahmjit Singh , "On the decision fusion for cooperative spectrum sensing in cognitive radio networks", Wireless Networks, pp. 2253 - 2262, 2017. https://doi.org/10.1007/s11276-0161285-0.

[18] Thuc Kieu-Xuan and Insoo Koo, "A Cooperative Spectrum Sensing Scheme Using Fuzzy Logic for Cognitive Radio Networks”, KSII TRANSACTIONS ON INTERNET AND INFORMATION SYSTEMS, vol. 4, no. 3, pp. 289 - 304, 2010. DOI: 10.3837/tiis.2010.06.006.

[19] Jaewoo So and Wonjin Sung, "Group-Based Multibit Cooperative Spectrum Sensing for Cognitive Radio Networks”, IEEE Transactions on Vehicular Technology, vol. 65, no. 12, pp. 10193 - 10198, 2016. DOI: 10.1109/TVT.2016.2536659.

[20] Junhai Luo and Xiaoting He, “A Soft-Hard Combination Decision Fusion Scheme for a Clustered Distributed Detection System with Multiple Sensors”, Sensor, vol. 18, 2018. DOI: 10.3390/s18124370.

[21] Yin Mi, Guangyue Lu, Yuxin Li and Zhiqiang Bao, “A Novel Semi-Soft Decision Scheme for Cooperative Spectrum Sensing in Cognitive Radio Networks", Sensor, vol. 19, no. 11, 2019. DOI: 10.3390/s19112522.

[22] Yuanhua Fu, Fan Yang and Zhiming He, “A Quantization-Based Multibit Data Fusion Scheme for Cooperative Spectrum Sensing in Cognitive Radio Networks”, Sensor Journal, vol. 18, no. 2, 2018. DOI: 10.3390/s18020473.

[23] Dongho Seo, Hyeongyun Kim and Haewoon Nam, "SDR Implementation of Energy Detection With Non-Uniform Quantization Scheme", in International Conference on Information and Communication Technology Convergence (ICTC), Jeju, South Korea, 2017. DOI: 10.1109/ICTC.2017.8190814.

[24] Huayan Guo, Nima Reisi, Wei Jiang and Wu Luo, "Soft Combination for Cooperative Spectrum Sensing in Fading Channels", IEEE Access, vol. 5, pp. 975 - 986, 2016. DOI: 10.1109/ACCESS.2016.2628860.

[25] Ibrahim Mustapha, Borhanuddin Mohd Ali, Mohd Fadlee A Rasid, Aduwati Sali and Hafizal Mohamad, "An Energy-Efficient Spectrum- 
Aware Reinforcement Learning-Based Clustering Algorithm for Cognitive Radio Sensor Networks", Sensor, vol. 15, no. 8, 2015. DOI: 10.3390/s150819783.

[26] Dost Muhammad Saqib Bhatt, Nasir Saeed and Haewoon Nam, "Fuzzy C-Means Clustering and Energy Efficient Cluster Head Selection for Cooperative Sensor Network", Sensor, vol. 16, no. 9, 2016, https://doi.org/10.3390/s16091459.

[27] Hong-Sam T. Le, Hung D. Ly and Qilian Liang , "Opportunistic Spectrum Access Using Fuzzy Logic for Cognitive Radio Networks Hong-Sam”, Springer, vol. 18, p. 171-178, 2011, https://doi.org/10.1007/s10776-011-0148-y.

[28] Sylwia Romaszko and Petri Mähönen, "Fuzzy Channel Ranking Estimation in Cognitive Wireless Networks", in IEEE Wireless Communications and Networking Conference (WCNC), Istanbul, Turkey, 2014. DOI: 10.1109/WCNC.2014.6952964.

[29] Giri Prasad Raman and Venkatesan Perumal, "Neuro-Fuzzy Based TwoStage Spectrum Allocation Scheme to Ensure Spectrum Efficiency in CRN-CSS Assisted by Spectrum Agent”, IET Circuits, Devices \& Systems, vol. 13, no. 5, pp. 637 - 646, 2019. DOI: 10.1049/ietcds.2018.5128.

[30] Vasudev Dehalwar, Akhtar Kalam, Mohan Lal Kolhe and Aladin Zayegh, "Compliance of IEEE 802.22 WRAN for Field Area Network in Smart Grid", in IEEE International Conference on Power System Technology (POWERCON), Wollongong, NSW, Australia, 2016. DOI: 10.1109/POWERCON.2016.7754046.

[31] Gyanendra Prasad Joshi, Srijana Acharya and Sung Won Kim, "FuzzyLogic-Based Channel Selection in IEEE 802.22 WRAN”, Information Systems, 2015. https://doi.org/10.1016/j.is.2014.05.009, vol. 48, pp. 327-332,

[32] Muhammad Waqas Khan and Muhammad Zeeshan, "Fuzzy Inference Based Adaptive Channel Allocation for IEEE 802.22 Compliant Smart
Grid Network”. Telecommunication Systems, vol. 72, p. 339-353, 2019. https://doi.org/10.1007/s11235-019-00570-y.

[33] Thompson Stephan, Fadi Al-Turjman, K. Suresh Joseph, Balamurugan Balusamy and Sweta Srivastava, "Artificial Intelligence Inspired Energy and Spectrum Aware Cluster Based Routing Protocol for Cognitive Radio Sensor Networks", Journal of Parallel and Distributed Computing, 2010, https://doi.org/10.1016/j.jpdc.2020.04.007, vol. 142, pp. 90-105.

[34] Mir Mehedi Ahsan Pritom, Sujan Sarker, Md. Abdur Razzaque, Mohammad Mehedi Hassan, M. Anwar Hossain and Abdulhameed Alelaiwi, "A Multiconstrained QoS Aware MAC Protocol for ClusterBased Cognitive Radio Sensor Networks", International Journal of Distributed Sensor Networks, 2015, https://doi.org/10.1155/2015/ 262871.

[35] Noor Salout, Faroq Awin, Esam Abdel-Raheem and Kemal Tepe, "Combined Fusion Schemes for Cluster-Based Spectrum Sensing in Cognitive Radio Networks", in IEEE International Symposium on Signal Processing and Information Technology (ISSPIT), Louisville, KY, USA, USA, 2018, DOI: 10.1109/ISSPIT.2018.8642636.

[36] V. C. Gungor and M. K. Korkmaz, "Wireless Link-Quality Estimation in Smart Grid Environments", International Journal of Distributed Sensor Networks, 2012, https://doi.org/10.1155/2012/214068.

[37] J. Wang, I.-R. Chen, J. J. Tsai and D.-C. Wang, "Trust-Based Mechanism Design for Cooperative Spectrum Sensing in Cognitive Radio Networks”, Computer Communication, vol. 116, pp. 90-100, 2018, https://doi.org/10.1016/j.comcom.2017.11.010.

[38] Ju Ren, Yaoxue Zhang, Qiang Ye, Kan Yang, Kuan Zhang and Xuemin Sherman Shen, "Exploiting Secure and Energy-Efficient Collaborative Spectrum Sensing for Cognitive Radio Sensor Networks", IEEE Transactions on Wireless Communications, vol. 15, no. 10, pp. 6813 6827, 2016. DOI: 10.1109/TWC.2016.2591006. 